\title{
Constraints on Low-Energy Effective Theories from Weak Cosmic Censorship
}

\author{
Baoyi Chen, ${ }^{1}$ Feng-Li Lin $\odot,{ }^{2}$ Bo Ning, ${ }^{3}$ and Yanbei Chen ${ }^{1}$ \\ ${ }^{1}$ Burke Institute of Theoretical Physics and Theoretical Astrophysics 350-17, California Institute of Technology, \\ Pasadena, California 91125, USA \\ ${ }^{2}$ Department of Physics, National Taiwan Normal University, No. 88, Sec. 4, Ting-Chou Road, Taipei 11677, Taiwan \\ ${ }^{3}$ College of Physics, Sichuan University, Chengdu, Sichuan 610064, China
}

(Received 27 June 2020; accepted 6 January 2021; published 22 January 2021)

\begin{abstract}
We examine the weak cosmic censorship conjecture (WCCC) for the extremal charged black hole in possible generalizations of Einstein-Maxwell theory due to the high-order corrections, up to fourthderivative terms. Our derivation is based on Wald's gedanken experiment to destroy an extremal black hole. We find that the WCCC no longer holds for all possible generalizations. Thus, the WCCC can serve as a new constraint to the high-order effective field theories. However, our constraint is independent of photon's self-interactions so that precision measurement of quantum electrodynamics cannot constrain the WCCC. For higher-dimension operators induced by the one-loop correction for the minimally coupled spinor and scalar to gravity, our constraint is satisfied.
\end{abstract}

DOI: 10.1103/PhysRevLett.126.031102

Introduction.-Even though the curvature singularity of a black hole is hidden behind the horizon, it might still be possible to throw charged or spinning matter into a black hole in particular ways that can destroy the horizon, revealing the singularity previously hidden inside. This kind of gedanken experiments was first proposed long ago by Wald [1] to test the so-called weak cosmic censorship conjecture (WCCC) [2], which asserts that the above gedanken experiments cannot succeed in order to prevent the singularity from being visible. Although the WCCC can be checked easily for extremal black holes, it is nontrivial to prove for near-extremal black holes [1,3] and for general forms of matter. Recently, significant progress for the general proof of the WCCC has been made by Sorce and Wald [4] who adopted a general relativistic formulation of the energy conservation which can work for general forms of matter obeying the null energy condition (NEC). In this way, they were able to avoid solving the complicated dynamical problems of the infalling matter involving the self-force effect, and succeeded to show that the WCCC holds for the black holes in Einstein-Maxwell theory, up to second order variation of the black hole's mass, charge, and angular momentum. Moreover, their method of examining the WCCC also provides a systematic framework for general theories other than Einstein-Maxwell.

One compelling reason to examine the WCCC for more general theories of gravity and electromagnetism is that the standard Einstein-Maxwell theory, which can be a good approximation at low energies, may need to be corrected at higher energies. In the low-energy effective field theory (EFT), these quantum corrections can leave low-energy relics in the form of higher-order derivative terms beyond Einstein-Maxwell terms, modifying the black hole solutions, as well as the relativistic laws of the energymomentum conservation. These terms may also make the WCCC fail. If we take the WCCC as a universal physical principle, then only those high-order EFTs that admit the WCCC should be accepted. This is in a similar spirit of using the weak gravity conjecture [5,6] which takes "gravity force is the weakest in nature" as a new physical principle to constrain the high-order EFTs [6].

By dimensional counting, the leading order correction to Einstein-Maxwell theory is photon's quartic self-interaction through a virtual scalar or spinor loop, which is a pure effect of quantum electrodynamics without involving gravity. Its Lagrangian density takes the form [7]

$$
L \propto c_{7} F_{\mu \nu} F^{\mu \nu} F_{\rho \sigma} F^{\rho \sigma}+c_{8} F_{\mu \nu} F^{\nu \rho} F_{\rho \sigma} F^{\sigma \mu},
$$

and the coefficients $c_{7}$ and $c_{8}$ can be well measured by experiments [9]. The next leading order corrections to the Einstein-Maxwell background is given by the gravitonphoton-photon interaction with a scalar or spinor loop. For the minimally coupled case, the one-loop effective actions for the Einstein-Maxwell background induced by spinors and scalars are given by $[10,11]$

$$
\begin{gathered}
L_{\text {spinor }} \propto 5 R F^{2}-26 R_{\mu \nu} F^{\mu \rho} F_{\rho}^{\nu}+2 R_{\mu \nu \rho \sigma} F^{\mu \nu} F^{\rho \sigma}, \\
L_{\text {scalar }} \propto-\frac{5}{2} R F^{2}-2 R_{\mu \nu} F^{\mu \rho} F_{\rho}^{\nu}-2 R_{\mu \nu \rho \sigma} F^{\mu \nu} F^{\rho \sigma},
\end{gathered}
$$

where we have neglected terms proportional to $\nabla^{\mu} F_{\mu \rho} \nabla_{\nu} F^{\nu \rho}$, as they do not appear in the metric equation of motion, thus having no effects on the black hole metric 
or our parameter bound. We will specifically check whether the two theories violate the WCCC later.

EFTs, black-hole solutions, and extremality condition.To demonstrate the power of the WCCC as a constraint to the EFTs, in this work we consider the most general quartic order corrections to Einstein-Maxwell theory, which is given by the following EFT action:

$$
I=\int d^{4} x \sqrt{-g}\left(\frac{1}{2 \kappa} R-\frac{1}{4} F_{\mu \nu} F^{\mu \nu}+\Delta L\right),
$$

where [12]

$$
\begin{aligned}
\Delta L= & c_{1} R^{2}+c_{2} R_{\mu \nu} R^{\mu \nu}+c_{3} R_{\mu \nu \rho \sigma} R^{\mu \nu \rho \sigma} \\
& +c_{4} R F_{\mu \nu} F^{\mu \nu}+c_{5} R_{\mu \nu} F^{\mu \rho} F_{\rho}^{\nu}+c_{6} R_{\mu \nu \rho \sigma} F^{\mu \nu} F^{\rho \sigma} \\
& +c_{7} F_{\mu \nu} F^{\mu \nu} F_{\rho \sigma} F^{\rho \sigma}+c_{8} F_{\mu \nu} F^{\nu \rho} F_{\rho \sigma} F^{\sigma \mu} .
\end{aligned}
$$

We will assume $c_{i}$ 's are small and restrict our consideration to $\mathcal{O}\left(c_{i}\right)$. The aforementioned self-interactions of four photons are the terms with coupling coefficient $c_{7}$ and $c_{8}$, respectively.

For simplicity, we will consider only the charged nonspinning black holes. The perturbative procedure of solving such black hole solutions has been outlined in [8], leading to a family of solutions parametrized by the mass and the charge $(M, Q)$. Here, we list some partial results relevant for our considerations (see Sec. I of Supplemental Material [14] for full expressions), namely the Maxwell gauge field

$A_{t}=-\frac{q}{r}+\frac{2 q^{3}}{5 r^{5}}\left[c_{5} \kappa+c_{6} \kappa\left(6-\frac{5 m r}{q^{2}}\right)+8 c_{7}+4 c_{8}\right]$,

and the $t t$ component of the metric (see Sec. II of Supplemental Material [14] for full expressions)

$$
\begin{aligned}
-g_{t t}= & 1-\frac{\kappa m}{r}+\frac{\kappa q^{2}}{2 r^{2}}+c_{2}\left(\frac{\kappa^{3} m q^{2}}{r^{5}}-\frac{\kappa^{3} q^{4}}{5 r^{6}}-\frac{2 \kappa^{2} q^{2}}{r^{4}}\right) \\
& +c_{3}\left(\frac{4 \kappa^{3} m q^{2}}{r^{5}}-\frac{4 \kappa^{3} q^{4}}{5 r^{6}}-\frac{8 \kappa^{2} q^{2}}{r^{4}}\right) \\
& +c_{4}\left(-\frac{6 \kappa^{2} m q^{2}}{r^{5}}+\frac{4 \kappa^{2} q^{4}}{r^{6}}+\frac{4 \kappa q^{2}}{r^{4}}\right) \\
& +c_{5}\left(\frac{4 \kappa^{2} q^{4}}{5 r^{6}}-\frac{\kappa^{2} m q^{2}}{r^{5}}\right) \\
& +c_{6}\left(\frac{\kappa^{2} m q^{2}}{r^{5}}-\frac{\kappa^{2} q^{4}}{5 r^{6}}-\frac{2 \kappa q^{2}}{r^{4}}\right) \\
& +c_{7}\left(-\frac{4 \kappa q^{4}}{5 r^{6}}\right)+c_{8}\left(-\frac{2 \kappa q^{4}}{5 r^{6}}\right)+\mathcal{O}\left(c_{i}^{2}\right) .
\end{aligned}
$$

Here, we define the reduced mass $m \equiv M / 4 \pi$, the reduced charge $q \equiv Q / 4 \pi$, and $\kappa=8 \pi G_{N}$, where $G_{N}$ is the gravitational constant. Note that in (7) there is no $\mathcal{O}\left(c_{1}\right)$ correction.

As shown by Ref. [8], as long as

$$
m \geq \sqrt{\frac{2}{\kappa}}|q|\left(1-\frac{4}{5 q^{2}} c_{0}\right),
$$

the singularity of the space-time will be hidden by a horizon; more precisely, the outer horizon located at the outermost solution of $g_{t t}\left(r_{H}\right)=0$. Here

$$
c_{0} \equiv c_{2}+4 c_{3}+\frac{c_{5}}{\kappa}+\frac{c_{6}}{\kappa}+\frac{4 c_{7}}{\kappa^{2}}+\frac{2 c_{8}}{\kappa^{2}},
$$

and $c_{0} \rightarrow 0$ recovers the Reissner-Nordstrom solution of Einstein-Maxwell theory. For a fixed $m$, as $q$ increases to, and then exceeds, the critical value at which equality holds in (8), two horizons will merge and subsequently disappear, revealing the singularity. In this way, the extremal solution is defined by imposing equality in (8). This implicitly defines a function $q_{\text {ext }}(m)$ for the extremal solution. For each $m$, the horizon radius of the extremal solution is given by

$$
r_{H}^{\mathrm{ext}}=\frac{m \kappa}{2}+\frac{4}{5 m}\left(c_{2}+4 c_{3}+\frac{10 c_{4}+c_{5}+c_{6}}{\kappa}-\frac{16 c_{7}+8 c_{8}}{\kappa^{2}}\right) .
$$

On this extremal horizon, the electrostatic potential is

$$
\Phi_{H}^{\mathrm{ext}}=-\left.\left(\xi^{a} A_{a}\right)\right|_{\mathcal{H}}=\sqrt{\frac{2}{\kappa}}\left(1+\frac{4 c_{0}^{\prime}}{5 q^{2}}\right),
$$

where $\vec{\xi}=\vec{\partial}_{t}$ is the timelike Killing vector of the spacetime, and

$$
c_{0}^{\prime}=-\frac{10 c_{4}}{\kappa}-\frac{2 c_{5}}{\kappa}-\frac{2 c_{6}}{\kappa}+\frac{4 c_{7}}{\kappa^{2}}+\frac{2 c_{8}}{\kappa^{2}} .
$$

Henceforth, we refer to $(m, q)$ solutions that strictly satisfy the inequality (8) as regular solutions, those that take equality as extremal solutions, and those that violate the inequality as singular solutions. We may still refer to them as "black holes," even though the horizon may or may not be destroyed.

Gedanken experiment to destroy the horizon.-In gedanken experiments that attempt to destroy the horizon, e.g., as set up by Wald $[1,4]$, we shall always (if tacitly) assume stability of our family of solutions. That is, starting off with a regular solution $(m, q)$, as we "throw matter into" it, the final space-time geometry and field configuration will settle down to another solution in our family. If our "way of throwing matter," for example, described by the on-shell metric perturbations, field perturbations, and matter stress-energy tensor in the initial slice, is 
parametrized by $w$, then the final solution should be given by $[m(w), q(w)]$.

In this language, the WCCC dictates that a starting regular solution $(m, q)$ long before "throwing matter" will only lead to $[m(w), q(w)]$ that are still regular. As a special case, let us now consider a starting extremal solution $\left[m, q_{\mathrm{ext}}(m)\right]$, and a particular approach of throwing matter, we can write

$$
\begin{aligned}
m(w) & =m+w \delta m+O\left(w^{2}\right), \\
q(w) & =q_{\mathrm{ext}}(m)+w \delta q+O\left(w^{2}\right) .
\end{aligned}
$$

The condition for the starting extremal solution to not become singular, at first order in $w$, is given by

$$
\delta m-\sqrt{\frac{2}{\kappa}}\left(1+\frac{4 c_{0}}{5 q^{2}}\right) \delta q \geq 0 .
$$

We therefore need to find out whether physical laws in our modified theory impose that (14) must hold for all infalling matter-or to find a particular way of throwing matter that violate (14). The advantage of starting off at the extremal solution is as follows: once Eq. (14) is violated, then any infinitesimal $w$ will lead to destruction of the horizon, and we can restrict ourselves to linear perturbation.

By contrast, starting from a nonextremal black hole with $\left[m, q_{\mathrm{ext}}(m)-\epsilon\right]$, a finite step size for $w$ must be made to surpass the extremality contour, and in this case the higher derivatives of $m(w)$ and $q(w)$ may become important, requiring the computation of higher-order variations. This was indeed the situation encountered by Hubeny [3], which was later addressed by Sorce and Wald [4] by considering the second order variations. Intuitively, one would expect the subextremal black holes will obey the WCCC if the extremal ones do, but the second order variations are needed for a rigorous examination on the subextremal case. In this Letter, we shall restrict ourselves to the extremal black holes.

As it turns out, condition (14) coincides with the requirement that the horizon area must increase as matter fall into extremal black holes (see Sec. IV of Supplemental Material [14] for details). More specifically, if we denote by $\mathcal{A}(m, q)$ the area of the horizon, then one can show that

$$
\partial_{m} \mathcal{A}(m, q) /\left.\partial_{q} \mathcal{A}(m, q)\right|_{q=q_{\mathrm{ext}}(m)}=d q_{\mathrm{ext}}(m) / d m,
$$

and that $d \mathcal{A}\left(m+w d m, q_{\text {ext }}(m)+w d q\right) / d w=0$ is equivalent to the equality in Eq. (14). In this way, the violation of condition (14), or the destruction of the extremal horizon, relies on the possibility of area decrease at linear order. This can be possible for the theories we consider even when the NEC is satisfied, because Raychaudhuri equation is now modified, and the NEC does not always lead to attractive gravity.
Test particle.-For a regular solution $(m, q)$, consider a test particle with reduced mass $\delta m_{0}$ and reduced charge $\delta q_{0}$, falling in from infinity. Using the minimally coupled action of

$$
S_{p}=4 \pi \int d \tau\left(\delta m_{0}-\delta q_{0} \vec{u} \cdot \vec{A}\right),
$$

the reduced canonical momentum of the particle, $\vec{p}=\delta m_{0} \vec{u}-\delta q_{0} \vec{A}$, satisfies $\vec{\xi} \cdot \vec{p}=$ const along the particle's trajectory; at linear order in $\delta m_{0}$ and $\delta q_{0}$, we do not have to consider the radiation reaction. Applying this to the particle at infinity and on the horizon, we obtain

$$
\delta m_{0}\left(\vec{u}^{H} \cdot \vec{\xi}\right)-\Phi_{H}^{c} \delta q_{0}=\delta m_{0}\left(\vec{u}^{\infty} \cdot \vec{\xi}\right)=-\delta E_{\infty},
$$

where $\vec{u}^{\infty}$ and $\vec{u}^{H}$ are the 4-velocities of the particle at infinity and on the horizon, and we have used the fact that $A_{t}$ does not depend on $t$, hence $\vec{\xi} \cdot \vec{A}$ vanishes at infinity.

For the final space-time, assuming that it still belongs to the same family, with $(m+\delta m, q+\delta q)$. We can argue from the charge conservation that $\delta q=\delta q_{0}$, and, from the conservation of ADM mass, as well as the fact that the energy of gravitational radiation emitted by the infall process is $\mathcal{O}\left(\delta m^{2}\right)$, that $\delta m=\delta E_{\infty}$ : basically, the charge and the energy of the particle are added to those of the black hole. We will soon give a more rigorous justification, but with this in hand we can write

$$
\delta m-\Phi_{H}^{c} \delta q=-\delta m_{0}\left(\vec{u}^{H} \cdot \vec{\xi}\right) \geq 0 .
$$

The latter inequality is because $\vec{u}^{H} \cdot \vec{\xi} \leq 0$ : the 4-velocity of the particle must be pointed toward the future as the particle crosses the horizon. This can be saturated if the particle is able to "rest right on top of the horizon." Inserting Eq. (11) into Eq. (18), we obtain the relation between $\delta q$ and $\delta m$ in this infalling test particle situation:

$$
\delta m \geq \sqrt{\frac{2}{\kappa}}\left(1+\frac{4 c_{0}^{\prime}}{5 q^{2}}\right) \delta q .
$$

This is clearly different from Eq. (14). However, before discussing its consequences, we shall introduce the framework by Sorce and Wald, which provides more rigorous treatment of the energy conservation, and is able to treat more general infalling matter.

Sorce-Wald method for generic matter.-We now sketch the method of Sorce and Wald developed in $[4,15]$. We follow the notation of Wald, and denote by $\phi=\left(g_{a b}, A_{a}\right)$ the metric and field degrees of freedom. We start off with an extremal black hole, with $\left[m, q_{\text {ext }}(m)\right]$, and define a Cauchy surface $\Sigma_{0}$ at early time, and a hypersurface $\Sigma_{1}$ which starts at sufficiently late time when the matter all fall in, and terminates at null infinity. We denote by $\mathcal{H}$ the portion of the extremal horizon between $\Sigma_{0}$ and $\Sigma_{1}$ (see Fig. 1). We then apply perturbation $\delta \phi$, as well as matter, with stress- 


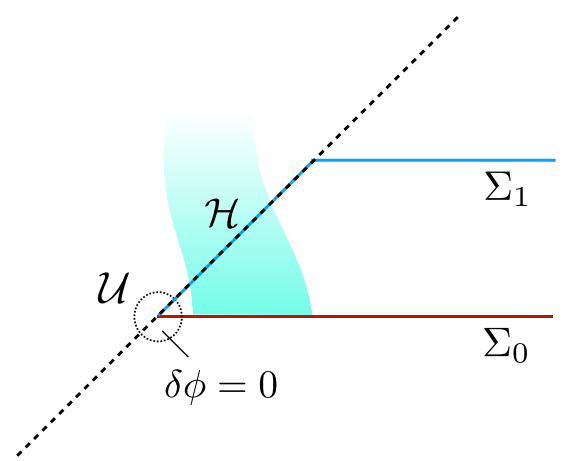

FIG. 1. The gedanken experiment to destroy an extremal black hole. Charged matter, occupying the shaded region, crosses the $\mathcal{H}$ portion of the extremal horizon.

energy tensor $\delta T_{a b}$ and electric current $\delta j_{a}$, also a form of perturbation, in an open neighborhood of $\Sigma_{0}$. We will set up our initial value problem in such a way that $\delta \phi, \delta T_{a b}$, and $\delta j_{a}$ all vanish in an open neighborhood $\mathcal{U}$ surrounding the intersection of $\mathcal{H}$ and $\Sigma_{0}$. In principle, $\delta \phi$ and $\delta T_{a b}, \delta j_{a}$ should be evolved jointly into the future, but here we assume stability of our family of solutions, and therefore can impose that space-time geometry in an open neighborhood of $\Sigma_{1}$ is that of $(m+\delta m, q+\delta q)$ [16].

A general Noether method to derive the law of energy conservation for such an infalling process is developed by Iyer and Wald [17], which we will briefly sketch as follows. Given a theory Lagrangian $L(\phi)$ of gravity and matter, we can introduce the Lagrangian 4-form $\boldsymbol{L}=L \boldsymbol{\epsilon}$, where $\boldsymbol{\epsilon}$ is the volume form associated with the metric. Then, variation of $\boldsymbol{L}$ yields

$$
\delta \boldsymbol{L}=\mathbf{E}(\phi) \delta \phi+d \Theta(\phi, \delta \phi),
$$

where $\mathbf{E}(\phi)=0$ is Euler-Lagrangian equation, and $\boldsymbol{\Theta}(\phi, \delta \phi)$ is the symplectic potential 3-form. For an arbitrary vector $\xi^{a}$, one can construct the associated Noether current $\mathbf{J}_{\xi}=\boldsymbol{\Theta}\left(\phi, \mathcal{L}_{\xi} \phi\right)-i_{\xi} \boldsymbol{L}$, which, because $\mathbf{J}_{\xi}$ is conserved, i.e., $d \mathbf{J}_{\xi}=0$, can be rewritten as $\mathbf{J}_{\xi}=$ $d \mathbf{Q}_{\xi}+\xi^{a} \mathbf{C}_{a}$ with the 3 -form constraint $\mathbf{C}_{a}=0$ when equations of motion are satisfied. For instance, in Einstein-Maxwell theory, the 3-form constraint is given by

$$
\left(\mathbf{C}_{a}\right)_{b c d}=\epsilon_{e b c d}\left(T_{a}^{e}+j^{e} A_{a}\right)
$$

with $T_{a b}=1 / \kappa\left(G_{a b}-\kappa T_{a b}^{\mathrm{EM}}\right)$ the nonelectromagnetic stress-energy tensor, and $j^{a}=\nabla_{b} F^{a b}$ the charge current of the Maxwell source. Thus the on-shell condition $\mathbf{C}_{a}=0$ gives the equations of motion $G_{a b}=\kappa T_{a b}^{\mathrm{EM}}$ and $\nabla_{b} F^{a b}=0$. The form (21) also holds when the higher-order derivative corrections $\Delta L$ are present. Assuming $\mathbf{E}(\phi)=0$ and $\xi^{a}$ is a Killing vector, i.e., $\mathcal{L}_{\xi} \phi=0$, it is easy to show that $\delta \mathbf{J}_{\xi}=d i_{\xi} \Theta(\phi, \delta \phi)$ which is then combined with $\delta \mathbf{J}_{\xi}=d \delta \mathbf{Q}_{\xi}+\xi^{a} \delta \mathbf{C}_{a}$, and is integrated over the hypersurface $\mathcal{H} \cup \Sigma_{1}$ to yield

$$
\int_{\infty}\left[\delta \mathbf{Q}_{\xi}-i_{\xi} \Theta(\phi, \delta \phi)\right]=-\int_{\mathcal{H} \cup \Sigma_{1}} \xi^{a} \delta \mathbf{C}_{a}
$$

where we have used the Stoke's theorem to turn the 3surface integral into the boundary integrals at the spatial infinity $\infty$ and at the intersection $\mathcal{H} \cap \Sigma_{0}$, by also imposing $\delta \phi=0$ at $\mathcal{H} \cap \Sigma_{0}$.

If we assume $\xi^{a}$ is the timelike Killing vector $t^{a}=\left(\partial_{t}\right)^{a}$ for nonspinning black holes, then we denote the change of the Arnowitt-Deser-Misner (ADM) mass as

$$
\delta \mathcal{M}=\int_{\infty}\left[\delta \mathbf{Q}_{\xi}-i_{\xi} \Theta(\phi, \delta \phi)\right]
$$

and the charge crossing the horizon as

$$
\delta \mathcal{Q} \equiv \int_{\mathcal{H}} \boldsymbol{\epsilon}_{a b c d} \delta j^{a},
$$

where the electric current $\delta j^{a}$ and the stress tensor $\delta T_{b}^{a}$ can be read off from the following on-shell relation [18]

$$
\left(\delta \mathbf{C}_{a}\right)_{b c d}=\boldsymbol{\epsilon}_{e b c d}\left(\delta T_{a}^{e}+A_{a} \delta j^{e}\right) .
$$

Combining all of the above and requiring the vanishing of $\delta j^{e}$ and $\delta T_{a}^{e}$ on $\Sigma_{1}$ as depicted in Fig. 1, we can turn (22) into the following law of energy conservation for the infalling process of Wald's gedanken experiment,

$$
\delta \mathcal{M}-\Phi_{H}^{c} \delta \mathcal{Q}=-\int_{\mathcal{H}} \boldsymbol{\epsilon}_{e b c d} \xi \xi^{a} \delta T_{a}^{e}
$$

On horizon $\mathcal{H}$ we can relate the 4-volume form $\boldsymbol{\epsilon}$ to the 3volume form $\tilde{\boldsymbol{\epsilon}}$ by the relation $\boldsymbol{\epsilon}_{e b c d}=-4 n_{[e} \tilde{\boldsymbol{\epsilon}}_{b c d]}$ where $n^{e}$ is the null vector normal to $\mathcal{H}$. Using this relation and the fact $\xi^{a} \propto n^{a}$ on $\mathcal{H}$, the right-hand side of (26) turns into $4 \int_{\mathcal{H}} \tilde{\boldsymbol{\epsilon}} \delta T_{a b} n^{a} n^{b}$, which is nonnegative if matter's stress tensor obeys the NEC. Thus, the variational identity (26) becomes an inequality for matter obeying the NEC,

$$
\delta \mathcal{M}-\Phi_{H}^{c} \delta \mathcal{Q} \geq 0 .
$$

This inequality serves as a constraint on the changes of the black hole's mass and charge for the infalling process, and will be used to check the WCCC by comparing with the condition (14).

Parameter bounds from WCCC.-The Noether method by Iyer and Wald provides a systematic way to calculate $\delta \mathcal{M}$ of (23) and $\delta \mathcal{Q}$ of (24) for general theory by evaluating $\boldsymbol{\Theta}, \mathbf{Q}$, and $\mathbf{C}_{a}$. For example, these quantities for EinsteinMaxwell theory have been derived in [17], and the results $\delta \mathcal{M}=4 \pi \delta m$ and $\delta \mathcal{Q}=4 \pi \delta q$ are then used to show that the WCCC holds for Einstein-Maxwell theory.

Here, we apply the same method for our higher-order theory (4). The derivation is tedious but straightforward, and the result is given in Sec. III of Supplemental Material 
[14], based on which we can evaluate the corresponding $\delta \mathcal{M}$ and $\delta \mathcal{Q}$. As a result, we find that $\delta \mathcal{M}=4 \pi \delta m$ because the corrections due to high-order Lagrangian $\Delta L$ fall off too quickly to contribute asymptotically to $\delta \mathcal{M}$. Similarly, we arrive $\delta \mathcal{Q}=4 \pi \delta q+\mathcal{O}\left(c_{i}^{2}\right)$ after tedious calculations [14]. The results are consistent with the test particle case. Therefore, we conclude that (27), which holds for general forms of matter obeying the NEC, gives the same condition Eq. (19) as for the test particle.

Compare the energy condition (19) and the WCCC condition (14), it is not hard to see that we must have $c_{0}^{\prime} \geq$ $c_{0}$ for the WCCC to hold for theory (4). Explicitly we have

$$
c_{2}+4 c_{3}+\frac{10 c_{4}}{\kappa}+\frac{3 c_{5}}{\kappa}+\frac{3 c_{6}}{\kappa} \leq 0
$$

This is our key result, which gives the parameter bounds on the low-energy EFT of quantum gravity by demanding that this low-energy theory preserves the WCCC.

Values of $c_{j}$ and connections to other bounds.-With our new bound (28) from the WCCC, it is then natural to ask how this bound works in the real world. Although black holes in the real world generally have nonzero angular momentum, our bound could still serve as a necessary condition. We first notice that the nonlinear EM terms contribute to $c_{7}$ and $c_{8}$, which do not appear in (28). This implies that the quantum electrodynamics automatically bypass the WCCC constraint. Plugging the values of $c_{4}, c_{5}$, and $c_{6}$ from the EFT (2) or (3) into the bound (28), we find the inequality also holds. An important implication is then, the WCCC not only holds for Einstein-Maxwell theory, but may also hold at one-loop level. This could possibly mean the correctness of the conjecture in the real world.

When the nonminimal coupling between matter and gravity is present, however, the bound (28) may subject to change under different situations. This is consistent with the fact that the combination of $c$ coefficients in our bound is not invariant under the field redefinition $g_{\mu \nu} \rightarrow g_{\mu \nu}+\delta g_{\mu \nu}$ [19], where

$\delta g_{\mu \nu}=r_{1} R_{\mu \nu}+r_{2} g_{\mu \nu} R+r_{3} F_{\mu \rho} F_{\nu}^{\rho}+r_{4} g_{\mu \nu} F_{\rho \sigma} F^{\rho \sigma}$.

With a proper choice of the matter-gravity coupling, it is even possible that there yields no bound for the $c$ coefficients, and that the WCCC always holds. A further discussion is beyond the scope of this Letter and we would like to explore it in the future.

It is interesting to compare our WCCC bound (28) with the bound obtained from the weak gravity conjecture (WGC), which is $[8,19]$

$$
c_{2}+4 c_{3}+\frac{c_{5}}{\kappa}+\frac{c_{6}}{\kappa}+\frac{4 c_{7}}{\kappa^{2}}+\frac{2 c_{8}}{\kappa^{2}} \geq 0 .
$$

Note that the WGC is a conjecture which states that gravity should be the weakest force for any consistent theory of quantum gravity. We see that the WGC bound will constrain $c_{7}$ and $c_{8}$ in contrast to the WCCC case. Moreover, these two bounds seem orthogonal to each other as one is bound above zero and the other bound below. Because of the seeming orthogonality, combing the WCCC and the WGC bounds together will be a useful tool to scrutinize the theory space of the high-order EFTs.

We thank Clifford Cheung for helpful discussions and comments. B. C. and Y. C. acknowledge the support from the Brinson Foundation, the Simons Foundation (Grant No. 568762), and the National Science Foundation, Grants No. PHY-1708212 and No. PHY-1708213. F. L. L. is supported by Taiwan Ministry of Science and Technology through Grant No. 106-2112-M-003-004MY3, and he also thanks NCTS for support. B.N. is supported by the National Natural Science Foundation of China with Grant No. 11975158.

[1] R. Wald, Gedanken experiments to destroy a black hole, Ann. Phys. (N.Y.) 82, 548 (1974).

[2] R. Penrose, Gravitational collapse: The role of general relativity, Riv. Nuovo Cimento 1, 252 (1969), https:// inspirehep.net/literature/54979; Gen. Relativ. Gravit. 34, 1141 (2002).

[3] V.E. Hubeny, Overcharging a black hole and cosmic censorship, Phys. Rev. D 59, 064013 (1999).

[4] J. Sorce and R. M. Wald, Gedanken experiments to destroy a black hole. II. Kerr-Newman black holes cannot be overcharged or overspun, Phys. Rev. D 96, 104014 (2017).

[5] C. Vafa, The string landscape and the swampland, arXiv: hep-th/0509212.

[6] N. Arkani-Hamed, L. Motl, A. Nicolis, and C. Vafa, The string landscape, black holes and gravity as the weakest force, J. High Energy Phys. 06 (2007) 060.

[7] We would expect that the Lagrangian density should be the linear combination of two parity-even and Lorenzinvariant terms, namely, $\left(F_{\mu \nu} F^{\mu \nu}\right)^{2} \propto \mathbf{E}^{2}-\mathbf{B}^{2}$ and $\left(F_{\mu \nu} \tilde{F}^{\mu \nu}\right)^{2} \propto \mathbf{E} \cdot \mathbf{B}$. Here, we follow the convention of [8] to replace the $\left(F_{\mu \nu} \tilde{F}^{\mu \nu}\right)^{2}$ term by another parity-even and Lorentz-invariant term $F_{\mu \nu} F^{\nu \rho} F_{\rho \sigma} F^{\sigma \mu}$.

[8] Y. Kats, L. Motl, and M. Padi, Higher-order corrections to mass-charge relation of extremal black holes, J. High Energy Phys. 12 (2007) 068.

[9] M. Bregant et al. (PVLAS Collaboration), Limits on low energy photon-photon scattering from an experiment on magnetic vacuum birefringence, Phys. Rev. D 78, 032006 (2008).

[10] F. Bastianelli, J. M. Davila, and C. Schubert, Gravitational corrections to the Euler-Heisenberg Lagrangian, J. High Energy Phys. 03 (2009) 086.

[11] F. Bastianelli, O. Corradini, J. Dávila, and C. Schubert, On the low-energy limit of one-loop photon-graviton amplitudes, Phys. Lett. B 716, 345 (2012).

[12] We have neglected terms proportional to $\nabla^{\mu} F_{\mu \rho} \nabla_{\nu} F^{\nu \rho}$, as it does not affect the black hole metric or our parameter bound. Further note that terms like $\left(\nabla_{\mu} F_{\nu \rho}\right)\left(\nabla^{\mu} F^{\nu \rho}\right)$ and $\left(\nabla_{\mu} F_{\nu \rho}\right)\left(\nabla^{\nu} F^{\mu \rho}\right)$ can be recast (up to some constant factor) 
into $\nabla^{\mu} F_{\mu \rho} \nabla_{\nu} F^{\nu \rho}$ plus existing terms in $\Delta L$ and an additional boundary term, upon using Bianchi identities, Ricci identities, and integrating by parts [13].

[13] S. Deser and P. van Nieuwenhuizen, One loop divergences of quantized Einstein-Maxwell fields, Phys. Rev. D 10, 401 (1974).

[14] See Supplemental Material at http://link.aps.org/ supplemental/10.1103/PhysRevLett.126.031102 for full explicit expressions and details. We arrive at $\delta \mathcal{Q}=4 \pi \delta q+$ $\mathcal{O}\left(c_{i}^{2}\right)$ after tedious calculations, as follows. By the construction of source theory, $j_{a}=\nabla^{b}\left(F_{a b}-S_{a b}\right)$ in which $S_{a b}$ is given in (5) of Supplemental Material and is of $\mathcal{O}\left(c_{i}\right)$, and using (3) of Supplemental Material, $F_{a b}=F_{a b}^{(0, j)}+S_{a b}+$ $\mathcal{O}\left(c_{i}^{2}\right)$ where the superscript $(0, j)$ means to evaluate by plugging the background Reissner-Nordström configurations and keeping up to $\mathcal{O}\left(c_{i}\right)$ terms. We then arrive $j_{a}=\nabla^{b} F_{a b}^{(0, j)}+\mathcal{O}\left(c_{i}^{2}\right)$, and use the Gauss's law the integral $\mathcal{Q}=\int_{\mathcal{H}} \boldsymbol{\epsilon}_{a b c d} j^{a}=\int_{B} * F^{(0, j)}+\mathcal{O}\left(c_{i}^{2}\right)$. Then, $\delta \mathcal{Q}=\int_{B}\left(* F^{(0, j)}-* F^{(0)}\right)+\mathcal{O}\left(c_{i}^{2}\right)=\delta Q+\mathcal{O}\left(c_{i}^{2}\right)$, where $\delta Q=4 \pi \delta q$ is the charge carried by the infalling matter. Thus, $\delta \mathcal{Q}=4 \pi \delta q+\mathcal{O}\left(c_{i}^{2}\right)$ is obtained.

[15] S. Hollands and R. M. Wald, Stability of black holes and black branes, Commun. Math. Phys. 321, 629 (2013).
[16] We note that in general $\Sigma_{1}$ is only a portion of a Cauchy surface - with the remaining portion completed by a portion of the future null infinity. A Cauchy surface that ends at spatial infinity does not approach $(m+\delta m, q+\delta q)$, fast enough; its ADM mass is not equal to $m+\delta m$ either, because it contains the energy-momentum content of gravitational waves emitted during the infall process; see, e.g., Ref. [15]. Sorce and Wald simply assumed that the late time solution is stable and nonradioactive to bypass the above concern [4]. On the other hand, in this Letter we consider only the infall of matter into an extremal black hole for which the dynamics is nonradioactive, thus the above issue does not exist in our consideration.

[17] V. Iyer and R. M. Wald, Some properties of Noether charge and a proposal for dynamical black hole entropy, Phys. Rev. D 50, 846 (1994).

[18] To arrive (25) we have imposed the on-shell conditions for the theory (4) along with the additional minimally coupled matter of stress tensor $\delta T^{a b}$ and charge current $\delta j^{a}$.

[19] C. Cheung, J. Liu, and G. N. Remmen, Proof of the weak gravity conjecture from black hole entropy, J. High Energy Phys. 10 (2018) 004. 